Anaesthesist 2009 $\cdot 58: 337-338$

DOI 10.1007/s00101-009-1544-x

Online publiziert: 8. April 2009

(c) Springer Medizin Verlag 2009

\author{
P. Knüfermann \\ Klinik und Poliklinik für Anästhesiologie und Operative Intensivmedizin, \\ Universitätsklinikum Bonn
}

\title{
Septisches Organversagen und zelluläre Fehlfunktion
}

den Übertragbarkeit der experimentellen Studienergebnisse auf den Menschen und führten z. T. sogar zu einer erhöhten Letalitätsrate [3]. Mitverantwortlich für den Misserfolg dieser älteren Studien war neben einer möglicherweise falschen Stratifizierung der Patientenkollektive auch der monokausale Therapieansatz mit fehlender Berücksichtigung von kompensatorischen antiinflammatorischen Mechanismen („,ompensatory anti-inflammatory response syndrome“, CARS; [4]) oder falsch gewählten therapeutischen Rahmenbedingungen [5]. Eine antiinflammatorische Intervention kann initial sinnvoll sein, um den autoaggressiven Charakter einer überschießenden Inflammation zu bremsen. Zu einem späteren Zeitpunkt kann diese Intervention $u$. U. eine Sepsis durch Kompromittierung der notwendigen Abwehrmechanismen zusätzlich aggravieren, die notwendigen Abwehrmechanismen kompromittieren und somit zu einer Schädigung des Wirtsorganismus beitragen.

Einen Meilenstein für ein besseres Verständnis der Komplexität der Immunantwort stellt die Entdeckung humaner Homologe von „Drosophila toll“ dar, einem Protein aus der Fruchtfliege mit essenzieller Bedeutung für die antiinfektive Antwort [6]. Die in Analogie zu Toll im Menschen beschriebene Familie der ,Tolllike"-Rezeptoren (TLR) ist als Vertreter der Mustererkennungsrezeptoren für die Fremd-Eigen-Diskriminierung mit hoher Spezifität verantwortlich. Die bisher beschriebenen TLR sind in der Lage, eine Vielzahl von pathogenen Liganden, z. B. bakterielle Wandbestandteile (Lipopolysaccharid, Lipoteichonsäure) oder bakterielle DNA zu erkennen und auf diesem
Weg eine Immunantwort $\mathrm{zu}$ initiieren. Von besonderem Interesse ist die Beobachtung, dass diese Rezeptoren nicht, wie ursprünglich vermutet, nur auf immunkompetenten Zellen nachgewiesen werden können, sondern auch von Gewebezellen in parenchymatösen Organen exprimiert werden [7]. Dies lässt die Vermutung zu, dass einzelne Organsysteme individuell auf einen inflammatorischen Stimulus regieren können, und ermöglicht eine neue Sichtweise auf das während der Sepsis häufig zu beobachtende Organversagen. Erste Arbeiten dokumentieren einen Zusammenhang zwischen organspezifischer TLR-Aktivierung und Organdysfunktion in verschiedenen murinen Modellen der Sepsis [8].

Der vorliegende Übersichtsartikel „Pathomechanismen des Organversagens Zelluläre Sauerstoffverwertungsstörung im Rahmen der Sepsis" von Wendel et al. [10] beschreibt aktuelle molekulare Erkenntnisse einer gestörten Zellfunktion und ihre Bedeutung für das Auftreten einer Organdysfunktion. Entzündungsmediatoren, ein vermehrter oxidativer Stress durch Produktion von „reactive oxygen species" (ROS) und reaktiven Stickstoffverbindungen („reactive nitrogen species“, RNS) sowie die Bildung von „nitric oxide“ (NO) schädigen die Aktivität und Integrität der mitochondrialen Enzymsysteme sowie der mitochondrialen DNA und besitzen somit direkten Einfluss auf die zelluläre Energiegewinnung. Insbesondere das hochreaktive Peroxynitrit schädigt Nukleinsäuren, Lipide und Proteine und inhibiert die zelluläre Atmungskette. Integrität und Funktion von Organen werden somit auf vielfältige Weise bis hin zum vollständigen Versagen be- 
einträchtigt; hierbei lässt sich die Ursache bis auf die subzelluläre Ebene zurückverfolgen. Eine mitochondriale Dysfunktion mit gestörter Energiegewinnung in der Atmungskette wurde im Skelettmuskel, in Hepatozyten und für das Krankheitsbild der akuten septischen Kardiomyopathie beschrieben.

Die von den Autoren beschriebenen Schädigungen der subzellulären Funktion sind letztendlich die Folge von Hypoxie, Inflammation und Dysregulation der Mikrozirkulation, denen eine zentrale Bedeutung im Verlauf von Sepsis und septischem Schock zukommt. Ein zu spätes Einsetzen der Therapie führt hier zum Multiorganversagen (MOV) und steigert die Letalitätsrate erheblich [9]. Die wichtigste Therapierationale ist deshalb die Rekrutierung von Kapillaren zur Wiederherstellung der Mikrozirkulation, um dem gestörten Gleichgewicht zwischen Sauerstoffangebot und Verwertung in der Zelle entgegenzuwirken. In der klinischen Praxis hat sich hierzu evidenzbasiert zum einen die frühzeitige und an hämodynamischen sowie metabolischen Zielen ausgerichtete Volumenersatztherapie durchgesetzt [2]. Zum anderen dienen eine Verbesserung des Herzminutenvolumens und/oder die Erhöhung des peripheren Gefäßwiderstands der Kreislaufstabilisierung. Beide Therapiekonzepte suggerieren, dass die Mikrozirkulationsstörung eine einfache Folge des systemischen Perfusionsdrucks ist. Ergebnisse aus der Grundlagenforschung zeigen aber, dass diese Sichtweise die tatsächlichen Vorgänge während einer Sepsis nur unzureichend abbildet [9]. Die Mikrozirkulation ist das Produkt einer großen Zahl an Interaktionen zwischen Endothel, Entzündungszellen und mikrohämodynamischen Veränderungen, die durch resultierende heterogene Blutflüsse das pathologische Bild eines distributiven Schocks verursachen. Wie von den Autoren des Übersichtsartikels gezeigt, ist dies mit komplexen intrazellulären Störungen vergesellschaftet.

Messungen von Größen in der Makrozirkulation (arterieller Mitteldruck, zentraler Venendruck oder pulmonal-kapillärer Verschlussdruck) verfehlen so das Ziel, den Schweregrad und Verlauf einer Sepsis verlässlich abzubilden. Auch die
Messung der $\mathrm{S}_{\mathrm{v}} \mathrm{O}_{2}$ als globalem Evaluationsparameter für die Zirkulation wird angesichts der Komplexität der inflammatorischen Matrix keine gute Hilfestellung für gezielte Eingriffe in den Entzündungsprozess leisten. Es stellt sich die Frage nach alternativen Diagnosekonzepten. Interessanterweise stehen bereits innovative Verfahren wie die orthogonale Polarisationsspektrometrie („OPS imaging“), die Laser-Doppler-Flowmetrie oder die sublinguale und submukosale Kapnographie zur Verfügung. Sie ermöglichen z. T. eine direkte Messung der Mikrozirkulation in der Klinik und werden zurzeit zur Evaluierung von Zielparametern für eine verbesserte Schockbehandlung eingesetzt. Ob eine breite Anwendung folgt, wird die Zukunft zeigen.

Wendel et al. [10] verdeutlichen mit ihrem Beitrag die komplexen zellulären $\mathrm{Me}$ chanismen, die das Bild der Sepsis prägen. Es ist allgemein anerkannt, dass pathogene Stimuli inflammatorische Signalwege aktivieren, die zum einen kaskadenartig innerhalb der Zelle verlaufen (vertikal) und zum anderen netzwerkartig mit weiteren Signalpfaden in Verbindung stehen (horizontal). Verkompliziert wird dies noch durch die Tatsache, dass die konsekutive Freisetzung von Entzündungsmediatoren organ- bzw. zellspezifisch verläuft und unterschiedlichen Zeitkinetiken folgt. Angesichts des wachsenden Wissens über die zahlreichen, z. T. parallel ablaufenden und sich gegenseitig beeinflussenden Mediatorsysteme wird es immer fraglicher, ob einzelne im klinischen Alltag gemessene Größen, die zurzeit als „Richtungsweiser“ für eine Therapie fungieren, das Stadium und den möglichen weiteren Verlauf der Inflammation realistisch abbilden können. Somit wird auch klar, warum monokausale Therapieansätze oder Strategien, die den Zeitverläufen der zu modulierenden Mediatoren nicht gerecht werden, vermutlich zum Scheitern verurteilt sind.

Eine der wesentlichen Aufgaben für die Zukunft wird es somit nicht nur sein, die molekularen Mechanismen der Sepsis zu beleuchten, sondern auch Hilfestellungen bei der Entwicklung von verfeinerten diagnostischen Werkzeugen und daraus abgeleiteten effizienteren Therapieansätzen zu leisten. Dazu benötigen wir zwei Din- ge ganz besonders: ein noch profunderes Wissen und eine enge Vernetzung von Wissenschaft und Klinik.

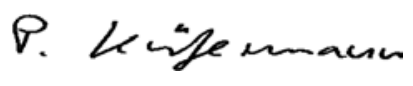

P. Knüfermann

\section{Korrespondenzadresse \\ PD Dr. P. Knüfermann}

Klinik und Poliklinik für Anästhesiologie und Operative Intensivmedizin, Universitätsklinikum Bonn Sigmund-Freud Str. 25Bonn

Pascal.Knuefermann@ukb.uni-bonn.de

Interessenkonflikt. Der korrespondierende Autor gibt an, dass kein Interessenkonflikt besteht.

\section{Literatur}

1. Engel C, Brunkhorst FM, Bone HG et al (2007) Epidemiology of sepsis in Germany: results from a national prospective multicenter study. Intensive Care Med 33:606-618

2. Rivers E, Nguyen B, Havstad S et al (2001) Early goal-directed therapy in the treatment of severe sepsis and septic shock. N Engl J Med 345:13681377

3. Natanson C, Hoffman WD, Suffredini AF et al (1994) Selected treatment strategies for septic shock based on proposed mechanisms of pathogenesis. Ann Intern Med 120:771-783

4. Bone RC, Grodzin CJ, Balk RA (1997) Sepsis: a new hypothesis for pathogenesis of the disease process. Chest 112:235-243

5. Abraham E (1999) Why immunomodulatory therapies have not worked in sepsis. Intensive Care Med 25:556-566

6. Anderson KV, Bokla L, Nusslein-Volhard C (1985) Establishment of dorsal-ventral polarity in the Drosophila embryo: the induction of polarity by the toll gene product. Cell 42:791-798

7. Baumgarten $G$, Knuefermann $P$, Schuhmacher $G$ et al (2006) Toll-like receptor 4, nitric oxide and myocardial depression in endotoxemia. Shock 25:4349

8. Knuefermann $P$, Schwederski $M$, Velten $M$ et al (2008) Bacterial DNA induces myocardial inflammation and reduces cardiomyocyte contractility: role of toll-like receptor 9 . Cardiovasc Res 78:26-35

9. Sakr Y, Dubois MJ, De BD et al (2004) Persistent microcirculatory alterations are associated with organ failure and death in patients with septic shock. Crit Care Med 32:1825-1831

10. Wendel M, Heller AR, Koch T (2009) Pathomechanismen des Organversagens - Zelluläre Sauerstoffverwertungsstörung im Rahmen der Sepsis. Anaesthesist \#:\#-\# 